\title{
Cerebral venous thrombosis: a rare presentation of paroxysmal nocturnal hemoglobinuria
}

\author{
H.K. Aggarwal ${ }^{1}$, Deepak Jain ${ }^{*}, 1$, Geeta Dabas ${ }^{1}$, R.K. Yadav $^{1}$, Promil Jain ${ }^{2}$ \\ ${ }^{1}$ Department of Medicine, Pt. B.D. Sharma University of Health Sciences, Rohtak (Haryana), India, \\ ${ }^{2}$ Department of Pathology, Pt. B.D. Sharma University of Health Sciences, Rohtak (Haryana), India
}

\begin{abstract}
Paroxysmal nocturnal hemoglobinuria (PNH) is a rare type of acquired hemolytic anemia characterized by persistent intravascular hemolysis often associated with pancytopenia and a tendency to venous thrombosis. The classical presentation is passage of hypochromic urine in morning along with hemolytic anemia, bone marrow failure and thrombophilia, but presentation as cerebral venous thrombosis is very rare. Here we present a case of 20 year old female with headache, seizure and hemiparesis, subsequently diagnosed with cerebral venous thrombosis (CVT) due to $\mathrm{PNH}$.
\end{abstract}

Keywords: paroxysmal nocturnal hemoglobinuria, cerebral venous thrombosis

\section{Introduction}

$\mathrm{PNH}$ is a rare acquired disorder of the hematopoietic system, characterized by increased susceptibility of red blood cells to complement mediated cell lysis, due to absence of glycophosphatidylinositol linked proteins.

It is characterized by a classical triad of acquired Coombs' negative intravascular hemolytic anemia, thrombophilia and various degree of bone marrow failure. The median age at disease onset is around 30 years, equally affecting men and women.

\section{Case report}

A 20 year old, 1st gravida, primipara, 3 months postpartum, female was admitted with complaints of abnormal involuntary

Received: November 2015; Accepted after review: December 2015; Published: December 2015.

${ }^{*}$ Corresponding author: Assist. Prof. Dr. Deepak Jain, Department of Medicine, Pt. B.D. Sharma University of Health Sciences, Rohtak-124001, (Haryana), India. Phone: +91-9416147887

Email: jaindeepakdr@gmail.com movements of entire body followed by loss of consciousness. There was no history of fever, vomiting, loose stools, severe headache, chronic cough, expectoration, weakness of any limb, trauma, bleeding or vaginal discharge, any drug intake or use of oral contraceptives. There was no history of similar episodes of seizures or blood transfusions. Her course of pregnancy was uneventful until 7 months period of gestation when she noticed decreased fetal movements; an abdominal ultrasound, was recommended, revealing an intrauterine fetal death; consequently, labor was induced, with delivery of the stillborn.

On physical examination, the patient appeared pale and jaundiced, afebrile, with a pulse rate of 90 per minute and blood pressure of $120 / 84 \mathrm{mmHg}$. On neurologic examination, the patient was in altered sensorium with a Glasgow Coma Score of 11/15, with preserved deep tendon reflexes and bilateral flexor plantars. No other changes were recorded regarding the physical examination.

The laboratory investigations revealed several pathological findings: hemoglobin was $6.7 \mathrm{~g} / \mathrm{dl}$ with mean corpuscular volume $98 \mathrm{fl}$, corrected reticulocyte count $3.5 \%$, but a 
normal platelet and leucocyte count. Patient's serum bilirubin was $4.2 \mathrm{~g} / \mathrm{dl}$ with indirect bilirubin of $3 \mathrm{~g} / \mathrm{dl}$, serum albumin was $4.2 \mathrm{~g} / \mathrm{dl}$ with normal liver transaminases and alkaline phosphatase levels. Macroscopic aspect and biochemical urine analysis were normal with no proteinuria or dysmorphic red blood cells (RBCs). Kidney function tests were normal. Serum lactate dehydrogenase (LDH) was markedly raised with a value of $2541 \mathrm{U} / \mathrm{L}$ $(<214 \mathrm{U} / \mathrm{L})$ and serum haptoglobin was $<20 \mathrm{mg} / \mathrm{dl}(30-200 \mathrm{mg} / \mathrm{dl})$. Serum vitamin B12 was $316 \mathrm{pg} / \mathrm{ml}$, folate level was $3.43 \mathrm{ng} / \mathrm{ml}$ and ferritin level was $57.4 \mathrm{ng} / \mathrm{ml}$ which were all normal. Direct and indirect Coombs' tests and Glucose-6-phosphate dehydrogenase (G-6PD) were negative. The test for osmotic fragility was normal, with hemolysis starting at $0.45 \%$ and completed at $0.35 \%$. Antinuclear antibodies were negative and protein $\mathrm{C}$, protein S, anti-thrombin III and antiphospholipid antibodies profile which were carried out 4 weeks afterwards were within normal limits, hence ruling out any coagulopathy. Hemoglobin electrophoresis was normal. Bone marrow analysis showed dimorphic erythroid hyperplasia with normal cellularity. Electrocardiography, chest X-ray and echocardiography disclosed no abnormalities. The abdominal ultrasound revealed no changes regarding the liver, gallbladder, pancreas, spleen and bilateral kidneys.

The noncontrast computer tomography (NCCT) of the head showed a hyperdensity with surrounding hypodensity located in the right high frontal region suggestive of bleed. Magnetic resonance imaging (MRI) demonstrated hemorrhagic infarct in right frontal lobe (Figure 1). MR venography showed non-visualization of superior sagittal sinus with visible thrombus (Figure 2).

In view of initial reports showing hemolysis and presence of cerebral sinus thrombus, a rare differential diagnosis of $\mathrm{PNH}$ was kept and flow cytometry was performed on granulocytes and RBCs, revealing that $95.6 \%$ granulocytes and $94 \%$ of RBCs were negative for CD55.

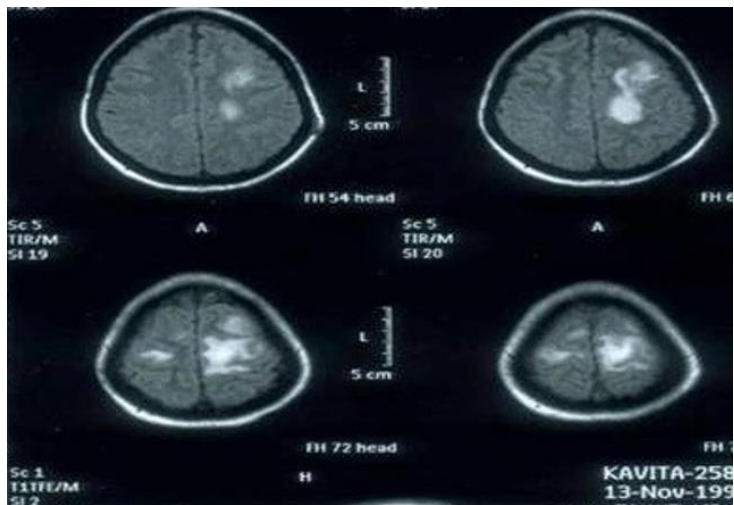

Fig. 1. Head MRI. Hemorrhagic infarct in right frontal lobe

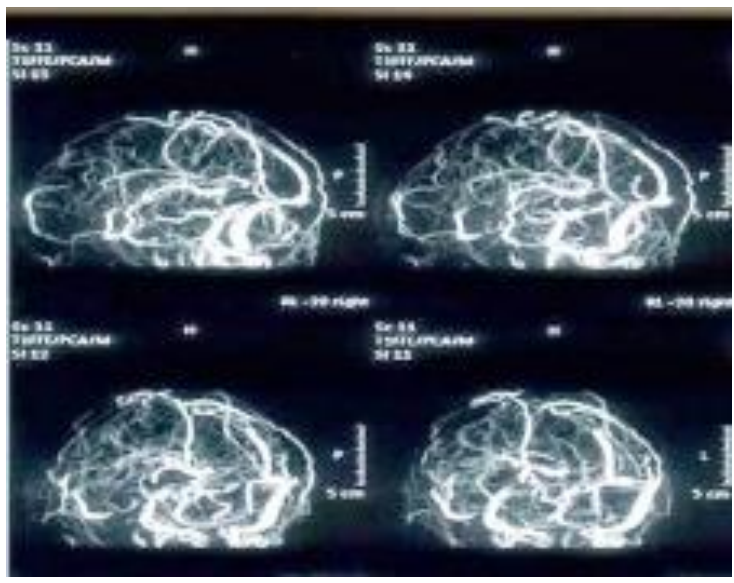

Fig. 2. MR venography. non-visualization of superior sagittal sinus with visible thrombus

Similarly $86.8 \%$ of granulocyte and $85 \%$ of RBCs were negative for CD 59 which confirmed the diagnosis of $\mathrm{PNH}$. Patient was started on Leviteracetam, heparin and warfarin and after an overlap period of 4 days, heparin use was stopped. Patient was discharged when the target INR was achieved on warfarin. Eculizumab therapy was recommended, but was refused by the patient, due to nonaffordability. In view of $\mathrm{PNH}$, Prednisolone (40 $\mathrm{mg}$ daily) was administered along with hematinic support. Patient was kept on regular follow up and her warfarin was stopped 3 months later; afterwards, her thrombophilia profile was re-evaluated and found to be normal. Patient's hemoglobin gradually improved and on last check-up, hemoglobin was $9.2 \mathrm{~g} / \mathrm{dl}$, total leucocyte count was $5000 / \mathrm{mm} 3$ with a platelet count of 3.0 lac. The 
serum bilirubin decreased to $2.0 \mathrm{mg} / \mathrm{dl}$ and $\mathrm{LDH}$ to $900 \mathrm{U} / \mathrm{L}$.

\section{Discussion}

$\mathrm{PNH}$ is a rare, chronic, debilitating disorder of hematopoietic system which usually presents with anemia. It is the only hemolytic anemia caused by an acquired intrinsic defect in the cell membrane i.e. the deficiency of glycophosphatidylinositol, leading to the absence of protective proteins on the membrane [1]. Reported incidence of clinically significant disease is in the range of 1 to 10 cases per million in population [2]. The median age of onset is around 30 years [3].

Patients with $\mathrm{PNH}$ present clinical features of unexplained hemolytic anemia like fatigue, jaundice and red colored urine. It can rarely present as thrombosis of arterial or venous system, which is the leading cause of death among patients with $\mathrm{PNH}$. Thrombosis occurs in up to $40 \%$ of patients of $\mathrm{PNH}$, but is rare as a first presenting symptom [4, 5]. The major risk factor for thrombosis appears to be the size of the PNH clone. The venous system is more frequently involved and the presentation depends on the site of thrombosis. The site of thrombosis in descending order of frequency is hepatic, pulmonary and mesenteric. However, sinus thrombosis as a presenting symptom is rarely described in literature.

$\mathrm{PNH}$ originates from an acquired genetic defect in a multipotent hematopoietic stem cell that acquires stem cell properties and is able to survive, expand, and self-renew. The acquired mutation in $\mathrm{PNH}$ occurs in the phosphatidylinositol glycan anchor biosynthesis, class A (PIGA) gene, which is responsible for the first step in the synthesis of the glycosylphosphatidylinositol (GPI) anchor that attaches a subset of proteins to the cell surface [6]. GPI anchors the protective proteins on surface of RBCs through peptide bond. Its deficiency leads to the loss of these protective proteins and makes the RBCs more susceptible to destruction by complement system.

The exact mechanism of thrombosis in $\mathrm{PNH}$ is unknown but various theories have been proposed. Platelet activation is likely to be the main culprit of high risk of thrombosis in $\mathrm{PNH}$. The absence of CD59 from platelets leads to thrombin generation, an increased sensitivity to aggregation by thrombin and increased risk of thrombosis. Activated platelets also interact with neutrophils and promote thrombus formation by release of serine proteases activating factor $X$ thus stimulating coagulation via extrinsic pathway. Another major contributor is endothelial damage caused by free hemoglobin and possibly complement system [7]. Nitric oxide (NO) plays an important role as platelet anti aggregator and in molecular regulation in coagulation cascade. So, the long-term consumption of NO by plasma hemoglobin has been implicated in the formation of clots in $\mathrm{PNH}$ patients [8]. Also, the increased procoagulant and fibrinolytic activity, suggesting increased fibrin generation and turnover, increased plasma levels of urokinase-type plasminogen activator and platelets deficient in GPI-linked proteins activated by complement have been implicated. Clinically, $\mathrm{PNH}$ is classified in three groups. The first one is the classical PNH which has the clinical evidence of intravascular hemolysis but no evidence of any other defined bone marrow abnormality. Second is $\mathrm{PNH}$ in setting of another specified bone marrow disorder. The last one is subclinical $\mathrm{PNH}$ with no clinical or laboratory evidence of hemolysis but only the presence of GPI deficient hematopoietic cells.

CVT is an uncommon disorder which presents with headache, seizure, focal neurological deficit, visual loss or rarely as encephalopathy. The most common predisposing factors are pregnancy, puerperium, infection and malignancy. Although our patient was in peripartum period which itself is a risk factor for thrombosis, the suspicion for other causes should also be kept in mind. All possible causes should be investigated before ruling out any treatable cause. PNH as a cause of CVT has been rarely described in literature. International experts in $\mathrm{PNH}$ suggest this disease should be tested for in all cases of CVT [9]. A high degree of suspicion can lead to early diagnosis and therapy initiation. 
Treatment of $\mathrm{PNH}$ is mainly supportive. Steroids are indicated if the process is chiefly hemolytic, but their long term use is limited by their toxicity. Iron is replaced if the patient has become iron deficient due to chronic blood loss in urine. Folic acid supplementation of $5 \mathrm{mg} /$ day is recommended to compensate the increased use. Cerebral sinus thrombosis in $\mathrm{PNH}$ is treated with adequate hydration, antiepileptic drugs for seizures and control of intracranial pressure. The antithrombotic therapy includes heparin, warfarin, thrombolysis and endovascular approaches. In case of $\mathrm{PNH}$, heparin followed by warfarin is used and the target INR is 2-3. The oral anticoagulation is continued for 3-6 months in CVT associated with PNH. Eculizumab, a humanized monoclonal antibody, has been found to be effective in treatment of $\mathrm{PNH}$. It acts by abrogating the complement mediated lysis and improves the quality of life of $\mathrm{PNH}$ patients. It is found useful in acute therapy of thrombosis in $\mathrm{PNH}$ but its role in cerebral venous thrombosis is not well defined and further trials are required to confirm its role and efficacy in such patients. It has also been found to reduce further thrombotic events in $\mathrm{PNH}$ [10]. Clinical data currently available indicate that Eculizumab has major impact in management of thrombosis in $\mathrm{PNH}$. Other

\section{References}

1. Kumar V, Abbas AK, Fausto N, Mitchell RN. Robbins Basic Pathology. $8^{\text {th }}$ Edition. Philadelphia: ElsevierSaunders; 2007.

2. Gulbis $B$, Eleftheriou $A$, Angastiniotis $M$, Ball $S$, Surralles $\mathrm{J}$, Castella $\mathrm{M}$ et al. Epidemiology of rare anemias in Europe. Adv Exp Med Biol 2010; 686:375-96

3. Schrezenmeier $H$, Muus $P$, Socié $G$, Szer J, Ispizua $\mathrm{A}$, Maciejewski $\mathrm{J}$ et al. Baseline characteristics and disease burden in patients in the International Paroxysmal Nocturnal Hemoglobinuria Registry. Haematologica 2014; 99:922-9.

4. $\mathrm{Pu} J \mathrm{~J}$, Brodsky RA. Paroxysmal nocturnal hemoglobinuria from bench to bedside. Clin Transl Sci 2011; 4:219-24.

5. Dacie JV, Lewis SM. Paroxysmal nocturnal haemoglobinuria: clinical manifestations, haematology, and nature of the disease. Ser Haematol 1972; 5:3-23. therapies like steroids and bone marrow transplantation have not been found to be effective in PNH associated thrombosis. There is a need to evaluate other therapies for such patients and to develop definitive guidelines for the use of anticoagulation to decrease future risks.

\section{Conclusion}

$\mathrm{PNH}$ is an uncommon cause of hemolytic anemia, which can rarely present as thrombotic episodes, such as cerebral venous thrombosis. The diagnosis- if thought of and sorted for early-gives us an opportunity to intervene hence, improving the patient related outcome.

\section{Consent}

Written informed consent was obtained from the patient for publication of this case report and accompanying images.

\section{Conflict of interest}

The author(s) declare that they have no competing interests.
6. Brodsky RA. Paroxysmal nocturnal hemoglobinuria. Blood 2014; 124:2804-11.

7. van Bijnen STA, Van Heerde WL, Muus P. Mechanisms and clinical implications of thrombosis in paroxysmal nocturnal hemoglobinuria. J Thromb Haemost 2012; 10:1-10.

8. Rother RP, Bell L, Hillmen P, Gladwin MT. The clinical sequelae of intravascular hemolysis and extracellular plasma hemoglobin: a novel mechanism of human disease. JAMA 2005; 293(13):1653-62.

9. Parker $C$, Omine $M$, Richards $S$, et al. Diagnosis and management of paroxysmal nocturnal hemoglobinuria. Blood 2005; 106:3699-709.

10. Hillmen $P$, Muus $P$, Duhrsen $U$, et al. Effect of the complement inhibitor eculizumab on thromboembolism in patients with paroxysmal nocturnal hemoglobinuria. Blood 2007; 110:4123-8. 